\title{
No bad thing
}

\author{
S. J. Hurley
}

Chief Dental Officer, England

The BDJ Upfront section includes editorials, letters, news, book reviews and interviews. Please direct your correspondence to the News Editor,

Kate Quinlan at BDJNews@nature.com. Press releases or articles may be edited, and should include a colour photograph if possible.

$\mathrm{C}$ hallenging and being challenged is no bad thing when making the case for overdue change. This year's Health and Care Innovation EXPO heralded Oral Health as an identifiable element of the programme. We exploited this opportunity to challenge healthcare colleagues to 'put the mouth back in the body' and include oral health in their care pathways.

EXPO also provided a platform for some commentators to regurgitate passé notions of dentistry. In challenging the hyperbole we offered evidence of 52 million patient contacts provided by the profession during the past year in support of NHS England, with two thirds of these patients exempt charges. We responded to the presumption of one size fits all six-month check-up by challenging existing patients to expect a bespoke recall interval based on their risk, lifestyle, and experience of disease. Despite the 12 years since NICE published their dental recall guidelines, the media considered the latter challenge a novel proposal with the result that the positives of the auditorium debate failed to make it into print.

The proposition is clearly not about discouraging regular attenders. However, the ardent patient conviction of an entitlement to a check-up every six months, irrespective of health risk, persists in some quarters. In yielding to these challenges we may limit practice capacity to accept new patients. Paradoxically, conversations with a small number of patients regarding their health risk and necessity of bi-annual recall appears to be underpinned by concerns that a practice will de-register them if they don't return every six months. I emphasise that this is patient perception not practice protocol. There are clearly patient and practitioner dilemmas to be resolved.

We should challenge patients with good oral health and low risk who demand a six month recall and many of us do. In questioning patient expectation we should not fear reprisal for extending recall interval to match risk; we are fulfilling the obligation to value and use health resources responsibly. Similarly, patients should feel comfortable verifying their recall interval, so they better understand what their level of dental health risk is and critically what they can do to reduce risk. At EXPO we offered patients licence to verify the rationale behind any repeated offer of a six month recall. The public accepts the necessity for enquiry in the course of their daily lives and the concept of trust but verify sits at the heart of the dynamic that maintains our partnership with our patients. These exchanges should not be seen as unusual. At the BDA Conference 2016 we accepted
Whilst I sense there is some existing capacity to implement the aspiration of Dental Checks by One Year for every child there remains the necessity to increase access in pursuit of improving oral health for all. In articulating our case for additional funded access, demonstrating that patients and practitioners are using current resources appropriately will be paramount. Our evidence base will also need to confirm best use of extant capacity in addressing health inequalities.

We also need to think ahead and anticipate the implications of reform within England and the transition to capitation. Practices garnering a resilient patient base, employing the care

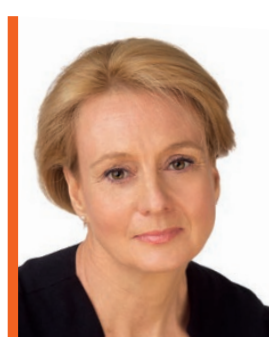

\section{'Are we certain that current access is being used responsibly?'}

government plaudits for our contribution to improving oral health and credit for supporting a growing proportion of the population in sustaining good oral health over the long term. Unsurprisingly, these health improvements come with an expectation of a dividend that lays bare new challenges for our profession. Detractors will point to enduring health inequalities; groups of individuals with limited success in accessing our care, statistics citing almost five million children not attending an NHS dentist in 2015/16. All reinforce a view that, amongst other health priorities, the dental access equation would benefit from some re-balancing. In challenging commissioners to secure greater access we must ask ourselves: Are we certain that current access is being used responsibly? Are there unidentified opportunities? How may the profession assist in re-aligning access? pathways advocated by the Steele review have successfully adopted the bespoke recall interval.

Changing public expectation today future proofs us for Contract Reform - no bad thing. It will assist us in securing funding for additional access - no bad thing. Challenging public expectation may be the best chance of ensuring the access to care that diverts a child from the caries pathway leading to the GA suite - no bad thing.

Challenge is a natural bed-fellow to progress. We have nothing to fear from the challenge of re-shaping patient expectation. The art is appreciating the context of challenge and the intent.

DOI: 10.1038/sj.bdj.2016.709

1. Health and Social Care Information Centre NHS Dental Statistics for England 2015/16 published 23 Sep 2016.

2. Department of Health, NHS dental services in England: an independent review led by Professor Jimmy Steele. London: Department of Health, 2009 gateway reference 11268 